\title{
Magnetic Loop Models: from Sun to Stars
}

\author{
M. Jardine, A. Collier Cameron, K. Wood \\ School of Physics and Astronomy, University of St Andrews, \\ North Haugh, St. Andrews, Fife, SCOTLAND KY16 9SS
}

\section{J.-F. Donati}

Laboratoire dAstrophysique, Observatoire Midi-Pyrénées, 14 Av. E. Belin, F-31400 Toulouse, France

\begin{abstract}
.
I review recent progress in determining the nature of the loop structures that form the coronae of solar-like stars. This progress has been driven by observational advances, in particular the new results from Xray satellites (Chandra and XMM-Newton) and the availability of surface magnetograms from Zeeman-Doppler imaging. It is now clear that stars that are similar to the Sun in mass, but which rotate more rapidly, have a very different magnetic field structure. Their surfaces are more heavily spotted, with spots appearing at all latitudes, extending all the way up to the rotation pole. Their coronae are correspondingly much brighter in X-rays, containing plasma that is hotter and denser than on the Sun. In addition, stellar coronae can support massive co-rotating prominences out to many stellar radii. Recent efforts in modelling these magnetic structures are now bringing together both the surface magnetograms and also the coronal X-ray emission. The resulting coronal loop models show complex loop structures on all scales, with much of the X-ray emission coming from high latitudes where is does not suffer rotational self-eclipse. The observed high densities and X-ray emission measures are a natural consequence of the high magnetic flux density at the surface. The stripping of the corona due to centrifugal effects at high rotation rates can also explain the saturation and supersaturation of X-ray emission with increasing rotation rates, and the recent observation of a high rotational modulation in a supersaturated star.
\end{abstract}

\section{Introduction}

Observations of the solar corona have, particularly over the last 10 years, shown very clearly the degree of complex and dynamic structure in the Sun's magnetic field. These observations, such as the wonderfully high-resolution images from TRACE have prompted a great deal of theoretical work in modelling solar magnetic loops. Corresponding studies of the coronae of solar-like stars have traditionally been hampered by the lack of such observations. Until recently, stellar loop models were constrained only by observations of the total emission measure 


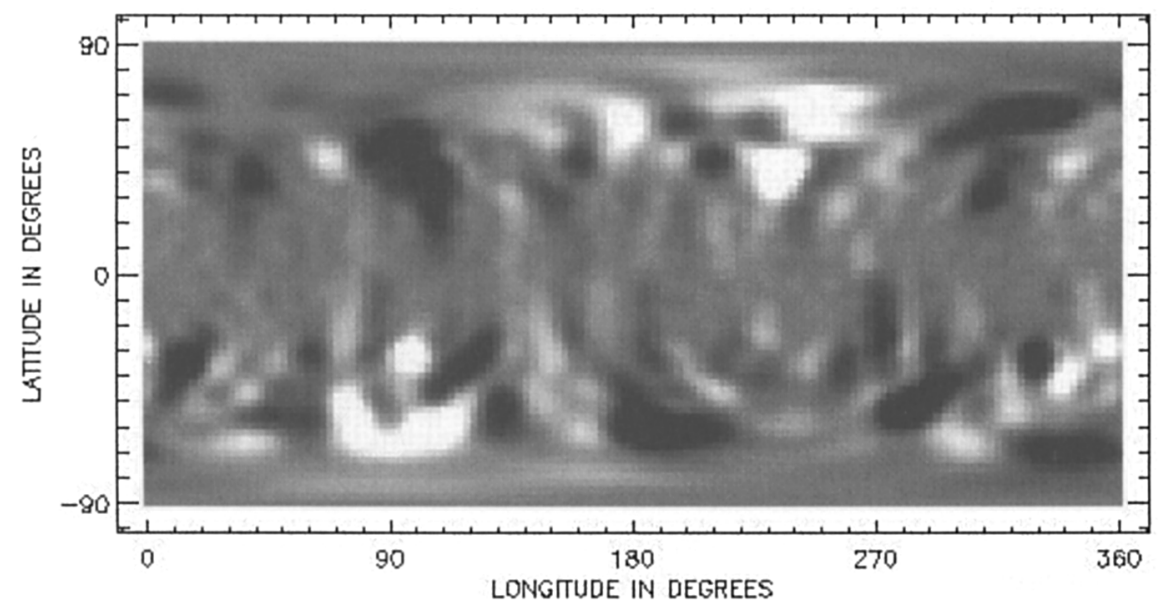

Figure 1. A map of the surface radial magnetic field of $A B$ Dor. White represents $-800 \mathrm{G}$ and black represents $800 \mathrm{G}$. Since AB Dor is inclined at $60^{\circ}$ to the observer, Zeeman-Doppler images provide only limited information in the lower hemisphere. In order to compensate for this, we have generated this combined surface map, with the 1995 map in the upper hemisphere and the 1996 map in the lower hemisphere.

of the star, or its rotational modulation. Over the last few years, however, this situation has changed dramatically. Advances in the areas of both X-ray satellite missions and in stellar surface imaging have provided much more information about the structure of stellar magnetic fields. In this review I will describe our current understanding of the nature of the loop structures in solar-like stars and compare them with their solar counterparts.

\section{Surface magnetic structures}

Solar-like stars of spectral types F-M are believed to generate their magnetic fields in a sub-surface dynamo within their convective regions. In the case of the Sun, this field is observed to emerge through the surface to form the familiar sunspot pattern. The behaviour of this pattern of flux emergence has been well studied over many magnetic cycles, with spots emerging first at mid latitudes (around $40^{\circ}$ ), then as the cycle progresses, emerging progressively closer to the equator (Schrijver \& Zwaan 2000). Coupled to this 11-year cycle in the spot pattern is a cyclic change in the structure of the large-scale coronal field, with the sign of the polar field reversing every 22 years. At solar minimum the largescale field is essentially dipolar, with higher-order modes increasing in strength through the cycle as more flux emerges through the surface.

In the case of stars that rotate sufficiently rapidly, this surface structure can be observed using Doppler imaging (Strassmeier 1996), or in the case of a few of the brightest stars, Zeeman-Doppler imaging which produces surface magnetograms (Donati \& Collier Cameron 1997). This has shown that stars of the same mass as the Sun, but rotating more rapidly, have a much greater 
spot coverage. The distribution of spots is also very non-solar, with spots at all latitudes, including the pole (see Fig. 1). While the appearance of polar spots in Doppler images indicates the presence of a polar field sufficiently strong to suppress convection, the nature of that field (whether unipolar or of mixed polarity) is as yet unknown. The Zeeman signature is suppressed in regions of the stellar surface that are dark and so Zeeman-Doppler maps typically contain little information on the nature of the polar field. If this field is of mixed polarity, it probably has little effect on the overall structure of the corona, but if it is unipolar it may significantly affect the large-scale structure (McIvor et al. 2003).

The detailed nature of any cyclic variation in spot patterns has yet to be determined, but in an interesting comparison with recent analysis of solar spot patterns (Berdyugina \& Usoskin 2003) there is mounting evidence for active longitudes on both single and binary stars (e.g. Jetsu et al. 1997; Korhonen et al. 2001). One rather surprising result from tracking the motions of starspots is that even very rapidly-rotating stars typically possess a latitudinal differential rotation that is very similar to the Sun's (Donati \& Collier Cameron 1997).

\section{Coronal magnetic structures}

Corresponding to the high coverage of surface field on these stars is a high $\mathrm{X}$ ray flux. Compared to the Sun, stars of increasing rotation rate show a rise in their X-ray emission that reaches a maximum of about $L_{x} / L_{b o l}=10^{-3}$ at rotation rates about $15-20 \mathrm{kms}^{-1}$ (Vilhu 1984). Beyond this rotation rate is the "saturated" regime where the X-ray luminosity is independent of rotation rate. These very active stars show frequent flaring, but no clear evidence of an $\mathrm{X}$-ray cycle has yet been determined. The Sun, on the other hand, although a much weaker X-ray source, shows variation of an order of magnitude in its X-ray flux over a cycle (Orlando, Peres, \& Reale 2000; Peres et al. 2000; Drake et al. 2000). It may be of course that these very active stars do indeed have a magnetic cycle whose X-ray signature is masked by the star's frequent and very powerful flares.

This "saturated" behaviour persists until rotation rates of about vsini > $100 \mathrm{kms}^{-1}$, where the X-ray luminosity begins to decrease again. This regime is referred to as "supersaturated" (Prosser et al. 1996; Randich 1998). There are several possible explanations for this variation with rotation rate. It may be that with increasing rotation rate, the dynamo process itself saturates due to the back-reaction of the field on the plasma. Alternatively the increasing dynamo activity may lead to a complete coverage of the stellar surface in active regions such that no further increase in X-ray emission is possible (Vilhu 1984). The explanation could also lie in the temperature range at which the coronae of rapid rotators are emitting. A shift to temperatures above the detection limit might produce an apparent saturation. Equally, the increase in the pressure scale height due to rapid rotation could lead to a greater fraction of cool magnetic loops in the corona and hence a shift to lower temperatures in the radiative output from the corona (Randich 1998).

A detection of rotational modulation of X-ray emission in a saturated star could potentially address some of these questions. A lack of any modulation would be consistent with a corona that is densely packed with X-ray emitting 
loops, while the presence of modulation might provide some clues as to which magnetic structures are dominating the emission. For many years, the lack of rotational modulation was taken as an indication of an extended corona. Eclipse mapping of binaries in particular suggested that the hotter component showed less modulation than the cooler component (White et al. 1990). This was not however a universally accepted view (see Giampapa et al. 1996; Siarkowski et al. 1996; Singh, White, \& Drake 1996) and the location and extent of the loops responsible for this emission was unclear (Jeffries 1998). A lack of rotational modulation could also be explained by placing the emitting regions in compact loops close to the rotation pole of the star where it would not be eclipsed.

A very clear example of this was seen in BeppoSAX observations of the decay of two flares on $\mathrm{AB}$ Dor $(\mathrm{P}=0.514$ days $)$. There was no rotational modulation of the X-ray emission over the decay phase of the flares, although they lasted for more than one rotation period. Modelling of the flare decay indicated that the flaring loops were small, with a maximum height of only $0.3 R_{\star}$. This implies that the flaring regions must have been located at latitudes above $60^{\circ}$ (the stellar inclination) where they were never eclipsed. This would also explain the low rotational modulation of only $5-13 \%$ seen in a long-term ROSAT study of AB Dor by Kürster (1997).

The idea that the high X-ray luminosity of very active stars comes not from a very extended corona, but from a much more compact set of solar-like active regions has also received support from line ratio studies. These suggested that coronal densities in the binaries Capella, $\sigma$ Gem and 44i Bootis are very high, perhaps up to $10^{13} \mathrm{~cm}^{-3}$ (Dupree et al. 1993; Schrijver et al. 1995; Brickhouse \& Dupree 1998). More recent observations of Capella by FUSE, Chandra and XMM-newton have also indicated high densities (Audard et al. 2001, Mewe et al. 2001, Young et al. 2001) (see also Güdel et al. (200)1 and Sanz-Forcada, Brickhouse, \& Dupree (2003) for XMM-Newton and EUVE results for a range of stars).

Measurements of coronal densities for AB Dor are also high (Maggio et al. 2000; Güdel et al. 2001). The Emission Measure Distribution as measured by both XMM-Newton and Chandra gives densities of around $\log n_{e}=10.8 \mathrm{~cm}^{-3}$ at $\log \mathrm{T}(\mathrm{K}) \approx 6.3$ and $\log n_{e}=12.5 \mathrm{~cm}^{-3}$ at $\log \mathrm{T}(\mathrm{K}) \approx 7$ (Sanz-Forcada, Maggio, $\&$ Micela, 2003). Its structure implies a corona composed of small $\left(\ell<R_{\star}\right)$ isobaric loops at different temperatures, whose filling factor is only $10^{-4}-10^{-6}$ of the stellar surface.

These studies of coronal densities and emission measures are, however, a very indirect way of determining the coronal structure. A much more straightforward technique is to use eclipse mapping as in the early work by White et al. (1990), Siarkowski et al. (1996) and Singh, White, \& Drake (1996). More recently, Güdel et al. (2003) have eclipse-mapped $\alpha \mathrm{CrB}$, a binary comprising an X-ray dark A0 star and a G5 V main-sequence "solar analogue" star. Their results show densities of $10^{9}-3 \times 10^{10} \mathrm{~cm}^{-3}$ and localised regions of emission on the G star, much of it at mid latitudes. This contrasts with the first "X-ray Doppler imaging" results from Chandra (Brickhouse, Dupree, \& Young, 2001). These implied that for $44 \mathrm{iBoo}$, at least half of the emission is localised at high latitude. 
Support for a highly complex, structured corona for active stars also comes indirectly from observations of so-called "slingshot" prominences (Collier Cameron \& Robinson 1987a,b). These are cool, dense clouds of mainly neutral hydrogen that are the stellar equivalent of solar prominences. They are observed as transient $\mathrm{H}_{\alpha}$ absorption features that cross the line profile on a timescale that depends on their distance from the rotation axis. They typically form at or just beyond the co-rotation radius (where gravity balances centrifugal forces) which for $A B$ Dor lies at $2.7 R_{\star}$ from the rotation axis. Since these prominences co-rotate with the star, at least over periods of a few days, they must be magnetically confined, even at these large distances. This places serious constraints on models of the stellar magnetic field.

\section{Modelling stellar coronae}

\subsection{Scaling up the rotation rate}

With the help of high resolution observations, solar physicists have made great strides in tackling fundamental problems such as the nature of the coronal heating mechanism. As described in other papers in this proceedings (see papers by Walsh and Aschwanden \& Title) there are still many unanswered questions. In particular the degree to which solar loops can be modelled as being hydrostatic or isothermal (e.g. recent papers by Schmelz (2002) and Aschwanden (2002)). In particular, it seems that modelling of single, static loops is being replaced by a "multi-thread" corona (Aschwanden \& Schrijver, 2002) composed of many fine-scale loops at different temperatures.

The long-standing question of course is to what degree we can take this understanding of solar loops and apply it to other stars. The correlation between the X-ray and radio fluxes in both solar flares and also in stellar (global) emission suggests that a single underlying mechanism is responsible for the energy release in both (Benz \& Güdel 1994). It appears that, on small scales, the magnetic field is releasing energy in the same way in the solar corona and in stellar coronae. On larger scales, however, there are significant differences between solar and stellar coronae that cannot simply be explained by scaling up the magnitude of solar activity.

These differences are apparent both in the surface distribution of flux and in the coronal structure and emission. The nature of the surface flux (which is often a lower boundary condition in loop models) is determined by the physics of field generation and transport in the sub-surface convective region. The principal factors determining the behaviour of the dynamo in this region are the stellar rotation rate and the differential rotation. The surface differential rotation is now being explored using (Zeeman)-Doppler imaging and is found to be similar to that for the Sun, even in very rapid rotators. This leaves the rotation rate itself as the prime factor determining the surface flux.

Once this flux erupts into the corona, its structure is determined both by conditions at its base and the gravitational stratification of the gas within it. In the equatorial plane, the gravitational acceleration is

$$
g=\left(-G M_{\star} / r^{2}+\omega^{2} r\right)
$$


where $\mathbf{g}=\mathbf{0}$ at the co-rotation radius,

$$
r_{K}=\left(G M / \omega^{2}\right)^{1 / 3} .
$$

For the Sun, the co-rotation radius is around $40 R_{\odot}$ and so we can safely ignore the effects of rotation in determining the coronal stratification. For higher rotation rates, however, such that the co-rotation radius is of order the extent of the corona, then rotation can have a significant effect on the coronal density structure. The principal effect is to lengthen the gravitational scale height, and also, for an isothermal plasma, to cause the plasma pressure to rise with height beyond the co-rotation radius. If this pressure becomes sufficiently high, it may distort the loop shape, or open it up entirely. The rise in density above the co-rotation radius may also be responsible for formation of prominences there.

In modelling stellar loops, then, the effects of rotation manifest themselves through the surface flux distribution (which may affect the ratio of open and closed field, the latitude of loops and their base pressure and field strength) and through the gravitational stratification of the coronal gas.

\subsection{Loop modelling}

As in the case of the Sun, much of the effort in modelling stellar loops in the past has focussed on modelling flare loops. Building on the early work of Rosner, Tucker, \& Vaiana (1978) and Serio et al. (1981), loop models were used to determine the length scale of flaring loops in an attempt to resolve the question of whether active stars have confined or extended coronae. The results were mixed and depended on the assumptions that were used. Thus, for example, the approach developed by van den Oord \& Mewe (1989) assumed a quasi-static decay phase and typically gave longer loop lengths - of order a stellar radius for Algol. The hydrodynamic approach developed by Reale (1997) however, suggested that sustained heating was indeed present during flare decay and produced estimates of loop sizes much smaller than a stellar radius (e.g. Favata, Micela, \& Reale 2001).

More recently, a new approach of modelling the whole corona has been developed. Schrijver (2001) (see also Schrijver 2003, this proceedings) developed a model of flux emergence and dispersal over the entire disk of a star. This showed that taking a solar model and simply increasing the rate at which bipoles emerge through the stellar surface produces the polar spots seen in Doppler images of rapidly rotating stars (Schrijver \& Title 2001) . In these models, flux emerges at low to mid latitudes (as observed on the Sun) and is carried to the poles by the meridional flow. Because the rate of emergence is higher than on the Sun, more flux reaches the polar cap before it is dispersed. An alternative explanation for high latitude flux on rapid rotators has been developed by Schüssler (1996) (see also Granzer et al. 2000). Flux generated in a dynamo at the base of the convective region is deflected poleward by Coriolis forces as it rises buoyantly to the surface.

This model produces a polar cap of mixed polarity, while the model of Schrijver \& Title (2001) produces a polar cap of a single polarity. This difference may become important when considering the implications of a particular stellar field model for the angular momentum evolution of young stars. Such stars are believed to spin down while on the main sequence due to the action of a hot, 

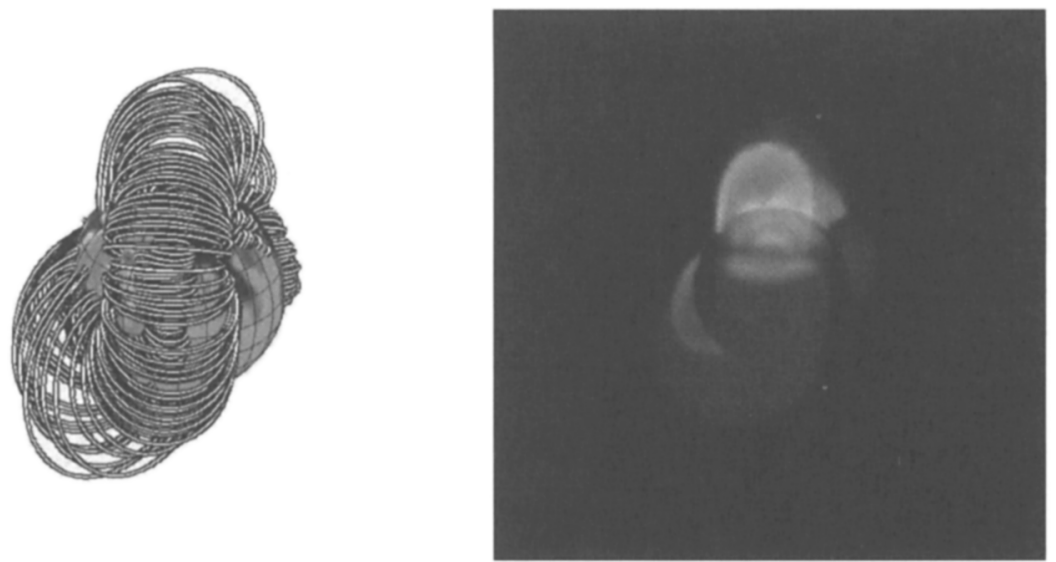

Figure 2. Field line extrapolations (left) and emission measure images (right) at temperatures of $10^{7} \mathrm{~K}$ for $\mathrm{AB}$ Dor in Dec. 2002.

magnetically-channelled wind. The amount of angular momentum carried away however depends on the field topology (McIvor et al. 2003, Schrijver, DeRosa, \& Title 2003). Schüssler \& Solanki (1996) demonstrated that a concentration of flux at high latitudes reduces the efficiency of angular momentum loss and may explain the distribution of spin rates in the young open clusters. Holzwarth \& Jardine (2003) have extended the traditional Weber-Davis wind model (Weber \& Davies 1967) to show that a non-uniform distribution of flux with latitude can have a significant impact on the angular momentum loss.

\subsection{Linking surface magnetograms to coronal X-ray emission}

Using their simulated surface magnetograms, Schrijver \& Aschwanden (2002) were able to extrapolate the potential field of the corona and model the X-ray emission of stars down to rotation rates of 5 days. Jardine, Collier Cameron $\&$ Donati (2002) used the surface magnetograms obtained by Zeeman-Doppler imaging of to extrapolate the potential coronal field of AB Dor. This work has also been extended to fit both potential and non-potential fields to the observed Stokes profiles (Hussain et al. 2001,2002). This technique allows us to explore which regions of the stellar surface show deviations from a potential field. In the case of $\mathrm{AB}$ Dor, these deviations are concentrated in the polar regions.

The potential field method of Jardine et al. (2002) is similar to that pioneered by Altschuler \& Newkirk (1969) and used by Wang et al. (1997) to model the solar green-light corona. Briefly, we write the magnetic field $\mathbf{B}$ in terms of a flux function $\Psi$ such that $\mathbf{B}=-\nabla \boldsymbol{\Psi}$ and the condition that the field is potential $(\nabla \times \mathbf{B}=\mathbf{0})$ is satisfied automatically. The condition that the field is divergence-free then reduces to Laplace's equation $\nabla^{\mathbf{2}} \mathbf{\Psi}=\mathbf{0}$ with solution in spherical co-ordinates $(r, \theta, \phi)$

$$
\Psi=\sum_{l=1}^{N} \sum_{m=-l}^{l}\left[a_{l m} r^{l}+b_{l m} r^{-(l+1)}\right] P_{l m}(\theta) e^{i m \phi}
$$



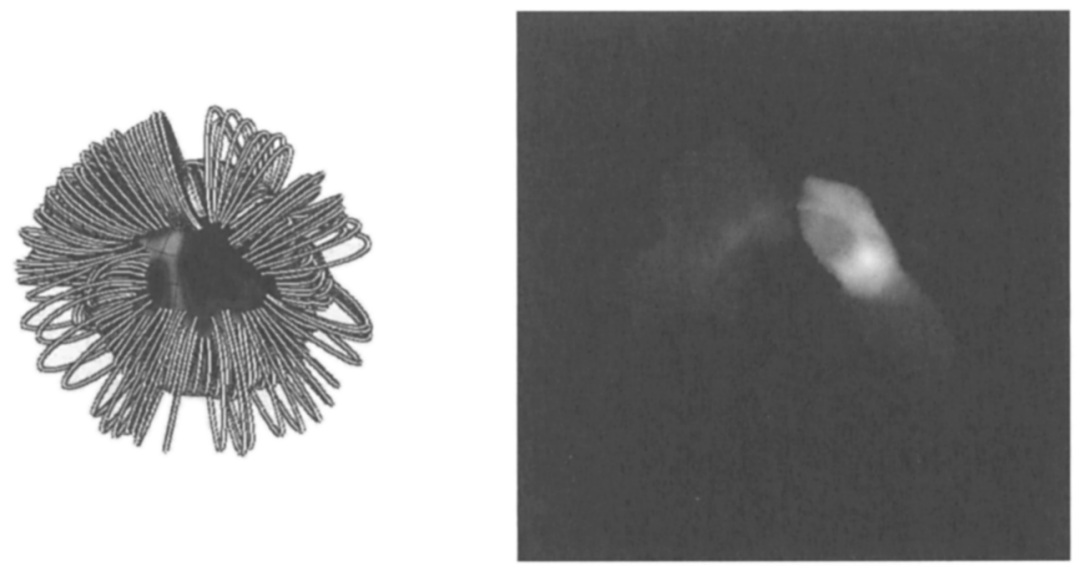

Figure 3. Field line extrapolations (left) and emission measure images (right) at temperatures of $10^{7} \mathrm{~K}$ for LQ Hya in Dec. 2000.

where the associated Legendre functions are denoted by $P_{l m}$. The coefficients $a_{l m}$ and $b_{l m}$ are determined by imposing the radial field at the surface from the Zeeman-Doppler maps and by assuming that at some height $R_{s}$ above the surface the field becomes radial and hence $B_{\theta}\left(R_{s}\right)=0$. This second condition models the effect of the plasma pressure in the corona pulling open field lines to form a stellar wind.

In order to calculate the X-ray emission, we must determine the coronal density structure. As a first step, we calculate the pressure structure of the corona assuming it to be isothermal and in hydrostatic equilibrium. Hence the pressure at any point is $p=p_{0} e^{\int g_{s} d s}$ where $g_{s}=($ g.B $) /|\mathbf{B}|$ is the component of gravity (allowing for rotation) along the field and

$$
g(r, \theta)=\left(-G M_{\star} / r^{2}+\omega^{2} r \sin ^{2} \theta, \omega^{2} r \sin \theta \cos \theta\right) .
$$

At the loop footpoints we scale the plasma pressure $p_{0}$ to the magnetic pressure such that $p_{0}(\theta, \phi)=R B_{0}^{2}(\theta, \phi)$ where $R$ is a constant. The plasma pressure within any volume element of the corona is set to zero if the field line through that volume element is open, or if the gas pressure exceeds the magnetic pressure (i.e. $\beta>1$ ) at any point along the field line. From the pressure, we calculate the density assuming an ideal gas and determine the morphology of the optically thin X-ray emission by integrating along lines of sight through the corona.

As an example of this method, Fig. 2 shows the field line extrapolation for $\mathrm{AB}$ Dor based on the Dec 2002 surface map (Donati private communication). The source surface has been placed at $3.4 R_{\star}$ since this is just beyond the positions at which prominences are observed to be confined by the coronal magnetic field. Shown are the largest-scale closed field lines. The global topology of the field is similar to that based on Zeeman-Doppler images from previous years. One side of the star is of dominantly positive polarity, while the other side is of dominantly negative polarity. The large scale field line connect these two regions, forming an arcade that runs right across the stellar 

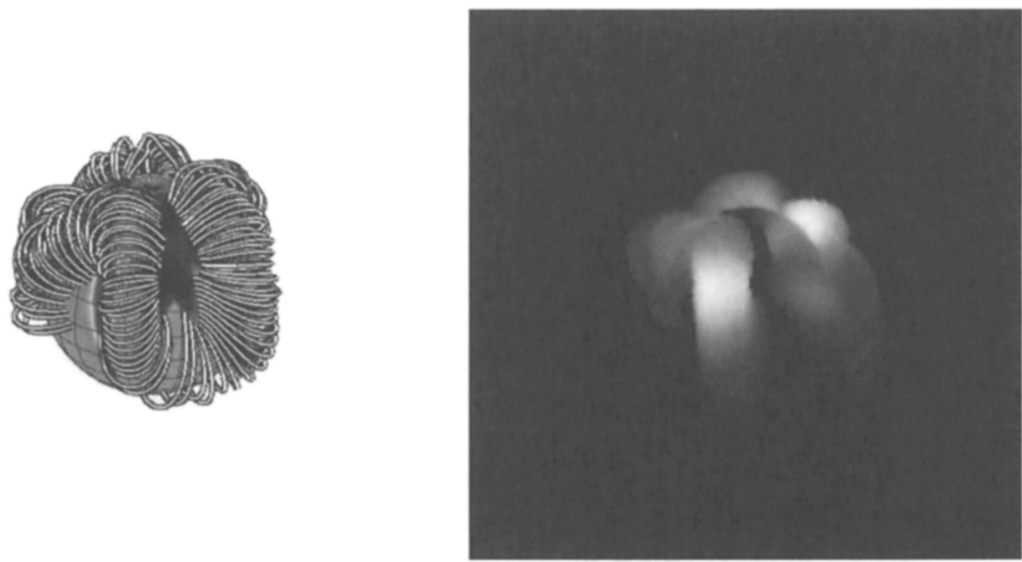

Figure 4. Field line extrapolations (left) and emission measure images (right) at temperatures of $10^{7} \mathrm{~K}$ for LQ Hya in Dec. 2001.

pole. This structure is also seen clearly in the emission-measure image. Since most of the brightest regions are at high latitude and so never pass behind the star, the rotational modulation is low $(\approx 5 \%)$. The maximum emission measure is $5.2 \times 10^{52} \mathrm{~cm}^{-3}$ and the emission-measure weighted density (defined as $\left.\int n_{e}^{3} d V / \int n_{e}^{2} d V\right)$ is $0.6 \times 10^{10} \mathrm{~cm}^{-3}$.

A similar field structure is seen in the LQ Hya ( $\mathrm{P}=1.6$ days) in Dec 2000 (Donati et al. 2003; McIvor et al. 2003). Again, there is a large-scale arcade that runs across the rotation pole (see Fig. 3). Significantly, however, the surface field maps show much more flux at low latitudes than on AB Dor and consequently there are more lower-latitude X-ray bright regions. Since these pass behind the star as it rotates, the calculated rotational modulation of $29 \%$ is much greater, although the maximum emission measure and density are similar $\left(3.4 \times 10^{52} \mathrm{~cm}^{-3}\right.$ and $0.6 \times 10^{10} \mathrm{~cm}^{-3}$ ) respectively.

The following year, however, the field structure of LQ Hya was significantly different (see Fig. 4). There were more changes of polarity around a line of latitude, perhaps indicating the emergence of a higher order mode. The images of the X-ray emission show several arcades running north-south, with once again most of the bright regions being at mid latitudes. The calculated rotation modulation of $33 \%$ was close to that of the previous year, however, as were the values of the maximum emission measure and density $\left(0.9 \times 10^{52} \mathrm{~cm}^{-3}\right.$ and $\left.0.4 \times 10^{10} \mathrm{~cm}^{-3}\right)$. It seems, therefore, that rotational modulation alone is not sufficient to distinguish different magnetic structures.

\subsection{Saturated and supersaturated stars}

The question of the nature of field structures in the most rapidly rotating stars has been brought to the fore very recently by the unambiguous detection of a $30 \%$ rotational modulation in the X-ray emission from VXR45, a very rapidly rotating supersaturated star in IC2391 (Micela et al. 2003). This is a dG9 star, with a rotation period of only 0.223 days. Doppler images (Marsden 2003) show it to be heavily spotted, with spots extending from low latitudes all the 

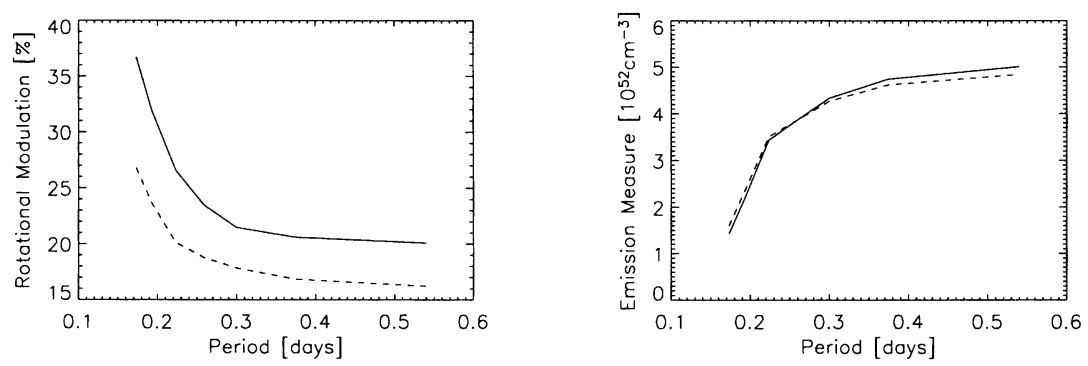

Figure 5. Shown left is the rotational modulation of the X-ray emission measure based on the magnetogram shown in Fig 1. Results are shown for two assumed stellar inclinations, $90^{\circ}$ (solid) and $60^{\circ}$ (dashed). Shown right is the corresponding emission measure.

way up to a dark polar cap. The high rotational modulation, however, suggests that a large proportion of the corona is dark, perhaps because the field lines are open. This observation is a conclusive demonstration that at least in this case, a supersaturated star does not have a corona that is filled to capacity with X-ray bright active regions.

This high rotational modulation is, however, a natural consequence of rotational stripping of the corona proposed by Jardine \& Unruh (1999) to explain the saturation and supersaturation of the X-ray emission. At high rotation rates the co-rotation radius (where centrifugal forces balance gravity) moves inside the $\mathrm{X}$-ray emitting corona. The rise of gas pressure in the summits of magnetic loops then breaks open these loops to form open field regions that are dark in X-rays. This reduction in the emitting volume initially balances the rise in the density of the corona to give a saturation of the X-ray emission, but eventually enough of the coronal volume has been forced open that the X-ray emission falls with rotation rate. As a result, at the highest rotation rates much of the corona is filled with open field and so there should be a significant rotational modulation in X-rays.

While this early paper used simple dipole and quadrupole models of the magnetic field to determine the coronal density structure and hence the emission measure, it is now possible to use the field extrapolations based on the observed surface magnetograms. Zeeman-Doppler images of VXR45 do not exist, but using a magnetogram of AB Dor as an example, Jardine (2003) has demonstrated that an increase in the rotation rate alone results in a decrease in the magnitude of the emission measure and an increase in its rotational modulation in a way that is consistent with the recent observation of VXR45 (see Fig. 5).

\section{Future prospects}

One of the most exciting new areas of research is the study of low mass and premain sequence stars. The relationship between rotation and activity in pre-main sequence stars is complicated by the possible presence of a disk. Such disks may 
be difficult to detect and may or may not be actively accreting. Intriguingly, Feigelson et al. (2003) find that pre-main sequence stars in the Orion Nebula Cluster display activity at less than the saturation level, independent of the presence or absence of disks. Flaccomio, Micela, \& Sciortino (2003) on the other hand, examine a range of clusters and find activity at the saturation level for those stars without disks, and below saturation for those stars with disks. The suppression of X-ray flux by the disk may be due to the disk/magnetosphere interactions altering the stellar coronal geometry. Alternatively, disk locking may lead to lower stellar rotation rates and hence lower activity levels. Higher $\mathrm{X}$-ray extinction due to the disk may also be to blame.

Clearly, the structure of the coronal field and the way it interacts with the disk is an important question for young stars. Similar questions arise in the case of stars at the low-mass end of the main sequence, which may, like the very young stars, be fully convective and therefore not capable of sustaining a solar-like interface dynamo. Nonetheless, Doppler imaging of $\mathrm{M}$ dwarfs (Barnes \& Collier Cameron 2001) show significant spot coverage on these stars and their $\mathrm{X}$-ray emission is not significantly different from that of higher mass stars. For the very lowest mass stars, however, the $\mathrm{L}$ and $\mathrm{T}$ dwarfs, a drop in $\mathrm{X}$-ray emission is seen. For these stars, the atmospheric temperature may be so low that the field and gas are no longer coupled (Mohanty et al. 2002). It is in these very extreme regimes that our understanding of the physics of stellar magnetic loops is stretched to its limits and it is perhaps here, for the very young and the very low-mass stars, that the greatest modelling effort is needed.

\section{REFERENCES}

Altschuler M. D., Newkirk, Jr. G. 1969, Solar Phys., 9, 131

Aschwanden M. J., Schrijver C. J. 2002, ApJS, 142, 269

Aschwanden M. J. 2002, ApJ, 580, L79

Audard M., Behar E., Güdel M., et al. 2001, A\&A, 365, L329

Barnes J. R., Collier Cameron A. 2001, MNRAS, 326, 950

Benz A. O., Guedel M. 1994, A\&A, 285, 621

Berdyugina S. V., Usoskin I. G. 2003, A\&A, 405, 1121

Brickhouse N., Dupree A. 1998, ApJ, 502, 918

Brickhouse N. S., Dupree A. K., Young P. R. 2001, ApJ, 562, L75

Collier Cameron A., Robinson R. D. 1989a, MNRAS, 236, 57

Collier Cameron A., Robinson R. D. 1989b, MNRAS, 238, 657

Donati J.-F., Collier Cameron A. 1997, MNRAS, 291, 1

Donati J.-F. et al. 2003, MNRAS, in press

Drake J. J., Peres G., Orlando S. et al. 2000, ApJ, 545, 1074

Dupree A., Brickhouse N., Doschek G. et al. 1993, ApJ, 418, L41

Favata F., Micela G., Reale F. 2001, A\&A, 375, 485

Feigelson E. D., Gaffney J. A., Garmire G. et al. 2003, ApJ, 584, 911

Flaccomio E., Damiani F., Micela G. et al. 2003, ApJ, 582, 398

Güdel M. et al. 2001, in ASP Conf. Ser. 234: X-ray Astronomy 2000. p. 73

Güdel M., Arzner K., Audard M., Mewe R. 2003, A\&A, 403, 155

Giampapa M., Rosner R., Kashyap V. et al. 1996, ApJ, 463, 707 
Granzer, Th. and Schüssler, M. and Caligari, P. and Strassmeier, K.G. 2000, A\&A, 355, 1087

Hussain G. A. J., Jardine M., Collier Cameron A. 2001, MNRAS, 322, 681

Hussain G. A. J., van Ballegooijen A. A., Jardine M., Collier Cameron A. 2002, ApJ, 575, 1078

Jardine M., Unruh Y. C. 1999, A\&A, 346, 883

Jardine M., Collier Cameron A., Donati J.-F. 2002, MNRAS, 333, 339

Jardine M. 2003, MNRAS, in press

Jeffries R. 1998, MNRAS, 295, 825

Jetsu L., Pohjolainen S., Pelt J., Tuominen I. 1997, A\&A, 318, 293

Korhonen H., Berdyugina S. V., Strassmeier K. G., Tuominen I. 2001, A\&A, 379, L30

Kürster M., Schmitt J., Cutispoto G., Dennerl K. 1997, A\&A, 320, 831

Maggio A., Pallavicini R., Reale F., Tagliaferri G. 2000, A\&A, 356, 627

Marino A., Micela G., Peres G., Sciortino S. 2003, IBVS, 5427, 1

Marsden S., Waite I. A., Carter B. D., Donati J.-F. 2003, in IAU 2003, Joint Discussion Meeting JD9

McIvor T., Jardine M., Collier Cameron A. et al. 2003, MNRAS, 345, 601

Mewe R., Raassen A., Drake J. et al. 2001, A\&A, 368, 888

Mohanty S., Basri G., Shu F. et al. 2002, ApJ, 571, 469

Orlando S., Peres G., Reale F. 2000, ApJ, 528, 524

Peres G., Orlando S., Reale F. et al. 2000, ApJ, 528, 537

Prosser C., Randich S., Stauffer J., Schmitt J. 1996, AJ, 112, 1570

Randich S. 1998, in Donahue, R. and Bookbinder, J., ed, 10th Cambridge

Workshop on Cool Stars, Stellar Systems and the Sun. p. 501

Reale F., Betta R., Peres G. et al. 1997, A\&A, 325, 782

Rosner R., Tucker W. H., Vaiana G. S. 1978, ApJ, 220, 643

Sanz-Forcada J., Brickhouse N. S., Dupree A. K. 2003, ApJS, 145, 147

Sanz-Forcada J., Maggio A., Micela G. 2003, A\&A, 408, 1087

Schmelz J. T. 2002, ApJ, 578, L161

Schrijver C. J., Aschwanden M. 2002, ApJ, 566, 1147

Schrijver C. J., Title A. M. 2001, ApJ, 551, 1099

Schrijver C. J., \& Zwaan C. 2000, Solar and Stellar Magnetic Activity. New York : Cambridge University Press, 2000. (Cambridge Astrophysics Series ; 34)

Schrijver C. J., DeRosa M. L., Title A. M. 2003, ApJ, 590, 493

Schrijver C., Mewe R., van den Oord G., Kaastra J. 1995, A\&A, 302, 438

Schrijver C. J. 2001, ApJ, 547, 475

Schüssler M., Caligari P., Ferriz-Mas A. et al. 1996, A\&A, 314, 503

Serio S., Peres G., Vaiana G. S. et al. 1981, ApJ, 243, 288

Siarkowski M., Prés P., Drake S., White N., Singh K. 1996, ApJ, 473, 470

Singh K., White N., Drake S. 1996, ApJ, 456, 766

Strassmeier K. 1996, in Strassmeier, K.G., Linsky, J.L., eds, IAU Symposium

176: Stellar Surface Structure. Kluwer, p. 289

van den Oord G. H. J., Mewe R. 1989, A\&A, 213, 245

Vilhu O. 1984, A\&A, 133, 117

Wang Y.-M. et al. 1997, ApJ, 485, 419

Weber E. J., Davis L. 1967, ApJ, 148, 217

Young P., Dupree A., Wood B et al. 2001, ApJ, 555, L121 